\title{
La « dynastie Friedel » : une grande lignée de scientifiques
}

Olivier Hardouin Duparc (olivier.hardouinduparc@polytechnique.edu)

Laboratoire des Solides Irradiés (UMR CNRS-CEA-X), École polytechnique, 91128 Palaiseau

Charles, Georges, Edmond, Jacques, et plusieurs autres : résumer la « dynastie scientifique Friedel » est une gageure que je tente néanmoins pour proposer au lecteur quelques jalons introductifs à cette lignée si célèbre dans le monde de la chimie, de la minéralogie, de la cristallographie, de la physique des solides et de la plasticité des matériaux.

\section{Jacques Friedel se considérait} lui-même comme une "graine de mandarin ", au meilleur sens du terme. Son décès l'an dernier, fin août 2014 à l'âge de 93 ans, son rôle actif en physique jusqu'à ses toutes dernières semaines, l'amitié qu'il m'accordait depuis plusieurs années déjà, et la fierté qu'il avait de sa grande famille justifient ces rappels.

\section{Les origines, jusqu'à Charles Friedel (1832-1899)}

Des Friedel alsaciens luthériens étaient tanneurs de père en fils jusqu'à ce que, à la faveur de la Révolution, l'un d'entre eux, prénommé Jean-Jacques, devienne négociant en cuir et épouse la fille d'un instituteur calviniste et organiste (les recommandations de Jean Calvin en la matière n'étaient plus considérées comme fondamentales). Ils mirent leur fils Charles au prestigieux Gymnase Jean Sturm de Strasbourg puis le marièrent à la fille du doyen de la faculté des sciences de Strasbourg, Georges Duvernoy. Charles (I) Friedel (1798-1882) s'établit commerçant comme son père mais en épicerie, puis créa une banque dont il espérait céder la direction à son fils Charles (II), à qui il avait également fait suivre ses études dans ce foyer humaniste libéral qu'était le Gymnase, fondé en 1538 et qui avait eu l'érudit et pédagogue Jean Sturm comme premier recteur.

Originaire de Montbéliard, Georges Duvernoy (1777-1855) était un lointain parent de Georges Cuvier (1769-1832), dont il sollicita l'aide et grâce à qui il fut nommé professeur à Strasbourg. Il devint son adjoint à Paris et finalement son successeur au Collège de France. Ce grand-père influença Charles (II), qui fit tout pour s'orienter vers les sciences alors que son père aurait voulu le voir entrer dans les affaires. Un cours de cristallographie de Pasteur à Strasbourg l'avait enthousiasmé, et il partit à Paris loger chez son grand-père dans l'ancienne maison de Buffon au Jardin des plantes pour préparer l'entrée à l'École normale. Un premier échec et l'obligation de signer allégeance à l'empereur Napoléon III en cas de succès l'orientèrent vers la faculté des sciences. Hésitant entre la cristallographie avec Pasteur et la chimie avec Charles-Adolphe Wurtz, il choisit Wurtz en 1854 tout en devenant attaché à la collection de minéralogie de l'École des mines de Paris (Mines ParisTech), sans doute en 1855, collection dont il fut nommé conservateur en 1856.

Son premier travail concerne les diamants (1856). C.F. approfondit entre autres, la connaissance de la chimie du silicium, basée sur sa tétravalence (on disait tétratomicité), montrant qu'elle était presque aussi riche que la chimie du carbone. C.F., comme Wurtz, était partisan de l'existence des atomes. Il découvrit surtout les réactions dites de Friedel et Crafts avec son grand ami américain francophile James Crafts en 1877, un peu par hasard mais tout en y étant parfaitement préparés. L'utilisation du chlorure d'aluminium comme catalyseur fut le coup de baguette magique pour les réactions sur les molécules d'hydrocarbures, si utiles plus tard tant dans l'industrie pétrolière que dans la parfumerie.

C.F. eut de nombreux élèves. Le père de Pierre Auger fit sa thèse dans son laboratoire de chimie et devint un professeur réputé à la Sorbonne. C.F. accueillit son lointain cousin Jacques Curie et étudia avec lui la pyroélectricité, en relation avec les propriétés de symétrie des cristaux et de leurs macles. Jacques et Pierre Curie découvrirent ensuite la piézoélectricité dans son laboratoire à la Sorbonne en 1880 (ce laboratoire devint ce qui est aujourd'hui, en 2015, l'Institut de minéralogie, de physique des matériaux et de cosmochimie, à Jussieu). 


\section{Le lignage scientifique Friedel / Crussard}

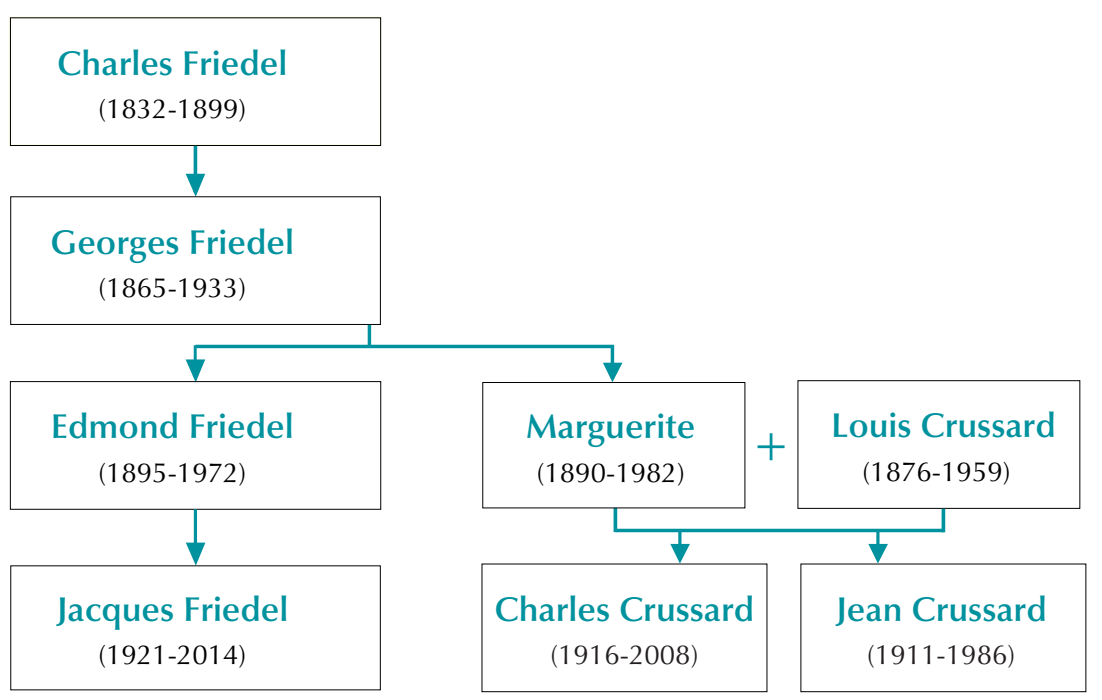

Suite à la défaite de 1870 et à la perte de son Alsace natale, C.F. contribua à la fondation de l'École alsacienne et créa le Laboratoire de chimie pratique et industrielle, destiné à former avec le même niveau scientifique à la fois des chimistes se destinant à l'industrie et des chimistes se destinant à la recherche, ainsi que le faisaient les universités allemandes. Cela devint l'École nationale supérieure de chimie de Paris (Chimie ParisTech). C.F. a été quatre fois président de la Société chimique de France qu'il avait aidée lors de sa naissance, de même qu'il avait contribué à la création de la Société française de minéralogie et de cristallographie. Outre son activité scientifique, Charles Friedel était membre de presque toutes les organisations protestantes de Paris. Il fut membre fondateur de la Ligue des droits de l'homme, l'année de son décès. Son dernier article décrivait un minéral radioactif, qu'il avait fait analyser par Pierre et Marie Curie.
1. Arbre généalogique simplifié et partiel, mais « synoptique ", de la « dynastie " Friedel. On n’y a pas indiqué Georges Duvernoy (1777-1855), qui est à la fois le grand-père de Charles Friedel (voir texte) et de Eugène Peugeot (1844-1907, fils de Jules, neveu d'Émile, cousin d'Armand avec qui il fit passer l'utilisation des aciers Peugeot des baleines de corsets aux bicyclettes puis aux voitures). Par la mère de Georges Friedel, on peut remonter jusqu'au mathématicien et physicien suisse Jean Bernoulli (1667-1748). L'un des frères ainés de Charles Crussard, Jean, avait commencé à travailler avec Louis Leprince-Ringuet sur la physique des rayons cosmiques. II dédia sa carrière à la physique des particules et à la physique nucléaire.
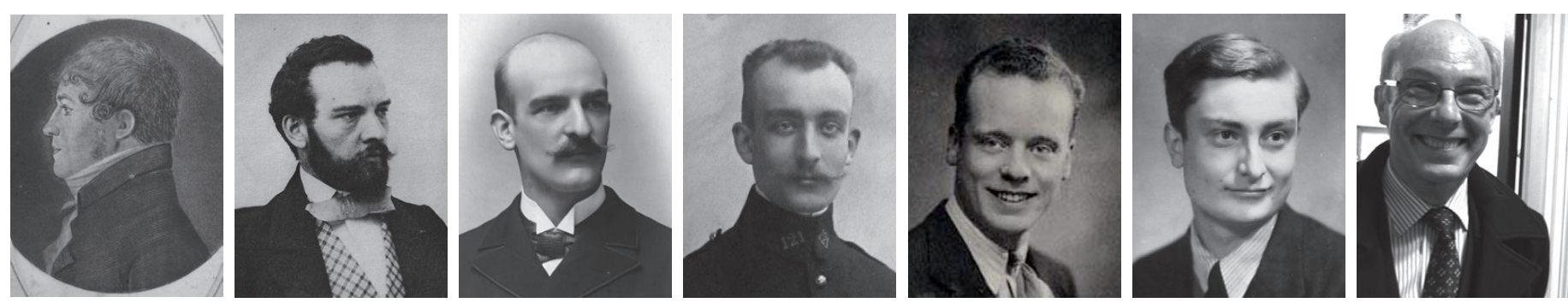

2. De gauche à droite : Georges (Duvernoy), Charles, Georges, Edmond, « Charlie » (Charles Crussard), Jacques et Paul. La belle barbe de l'aïeul Charles est d'origine « post quarante-huitarde », suite à la Révolution française de 1848. Je remercie grandement Jean Friedel pour son aide précieuse pour toutes ces photos et ce montage. 


\section{\> \\ De Charles Friedel à Georges Friedel (1865-1933)}

En 1896, C.F. se vit refuser par l'École polytechnique ( 1 l'X») le poste de professeur de chimie, tout comme Henry Le Chatelier. C.F. reprit la chaire de Wurtz à la Sorbonne, et son fils Georges intégra l'X l'année suivante en position de major. Doué en tout, y compris les sports et la musique, G.F. aurait voulu choisir ensuite le génie maritime mais, étant également sorti major, il dut intégrer le corps des mines et rejoignit ainsi son père avec qui il se spécialisa en minéralogie et cristallographie.

G.F. établit dès 1904 une classification toujours en vigueur des macles, avec le concept mathématique de réseau de coïncidence entre les grains caractérisé par un index de coïncidence, tout en sachant bien dès l'origine que "le réseau le plus intéressant à connaître pour expliquer les macles [est] le réseau matériel ", mais à l'époque ce réseau atomique était inconnu pour tous les minéraux. Il s'intéressa de près à la découverte de la diffraction par les cristaux en 1912 et il montra immédiatement que les diagrammes de Laue de l'époque, les " radiogrammes ", devaient être centrosymétriques et ne pouvaient donc révéler l'éventuelle hémiédrie d'un cristal (ce que l'œil nu peut faire à partir de l'examen des faces d'un cristal hémièdre). C'est ce qu'on appelle la « loi de Friedel ». G.F. étudia aussi dès 1910 les étranges matériaux de l'Allemand Otto Lehman, les fameux cristaux liquides, une appellation bien malgré lui mais entérinée par Charles Mauguin en 1911 et plus parlante que " états mésomorphes de la matière ". Après un certain scepticisme, G.F. en fit une étude magistrale en 1922 en les divisant en smectiques, nématiques et cholestériques.

G.F. dirigea l'École des mines de SaintÉtienne jusqu'en 1919, avant de rejoindre l'université de Strasbourg juste libérée. Il y professera ses leçons de cristallographie, sans jamais être reconnu comme professeur, parce qu'il n'avait pas de diplôme universitaire. Le corps des mines continuait à assurer son salaire. Dans toutes les disciplines où G.F. s'illustra, son antigermanisme, sans doute explicable par l'histoire de l'Alsace vue du côté français, s'exprimait autant que possible et sans ménagement. Sa fille Marguerite épousa en 1909 Louis Crussard (1876-1959, major de l'X), professeur à Saint-Étienne avant d'aller participer à la fondation de l'École des mines de Nancy en 1920.

\section{Edmond Friedel (1895-1972), Charles Crussard (1916-2008)}

Edmond Friedel fut également major de l'X et corps des mines. Il travailla avec Maurice de Broglie, frère aîné de Louis, sur les smectiques en 1925, utilisant les rayons $\mathrm{X}$ pour préciser des mesures de Jean Perrin et de son étudiant Wells, obtenues en lumière visible. Ils montraient que les lames minces de savon sur lesquelles Perrin avait découvert sa loi des épaisseurs multiples étaient des lames de corps smectique, ce qui confirmait ce que prévoyait G.F. en 1922, à savoir que les corps smectiques se composent bien de couches moléculaires équidistantes. E.F. s'occupa ensuite surtout de géologie pour la recherche de ressources minières, comme par exemple le pétrole, de manière hélas infructueuse au Maroc. Il dirigea l'École des mines de Paris. Outre l'enseignement d'économie qu'il y réorganisa après la guerre, il lança en 1939 avec le soutien d'Albert Portevin un Centre de recherches métallurgiques dont le premier directeur fut son neveu Charles Crussard (1916-2008, encore un major de l'X).

C.C. travailla beaucoup sur ce qu'il appela la recristallisation in situ dans les métaux, surtout l'aluminium, et devint directeur scientifique du centre de recherche du groupe industriel Pechiney à Voreppe, qu'il a contribué à créer après avoir participé au développement de l'Institut de recherches de la sidérurgie française (IRSID) à Saint-Germain-en-Laye.

\section{Jacques Friedel (1921-2014)}

L'épouse d'E.F., Jeanne, née Bersier, contracta la tuberculose probablement en 1923 et resta de santé fragile, nécessitant de fréquents séjours en montagne. Leur fils ainé Jacques Friedel reçut une éducation familiale et par correspondance jusqu'à l'âge de 10-11 ans. Lui et sa jeune sœur pouvaient ainsi accompagner leur mère autant que possible lors de ses séjours dans les Alpes ou dans le Jura. Grand maigre dégingandé, J.F. en garda un caractère plutôt timide et intériorisé. Il reçut une éducation très classique (mandarinale, écrira-t-il en 1994), avec latin, grec et allemand. Il apprit le grec avec sa tante Marie Friedel qui avait, dit-on, soufflé à son propre père les noms de ses états mésomorphes (smectique, nématique, cholestérique). Arrivé au baccalauréat, ses parents lui offrirent une année d'études classiques en philosophie et littérature (sans doute surtout à l'instigation de sa mère), avant une année scientifique. Balloté par les années de guerre, J.F. échoua deux fois à l'X et ne fut reçu " que " quatrième la troisième fois. Il se rattrapa brillamment par la suite.

Après une première expérience de recherche peu gratifiante comme stagiaire chez Louis Leprince-Ringuet à l'X, il alla travailler sur les joints de grains métalliques chez son cousin Charles Crussard qui l'envoya ensuite se spécialiser chez Nevill Mott à Bristol. Mott l'aiguilla sur le problème de l'intensité de l'écrantage de la charge d'une impureté par les électrons libres du métal, problème qu'il avait luimême étudié à l'aide d'un modèle qu'il savait être sans doute trop simple. J.F. s'y fit une réputation en montrant que cet écrantage décroissait moins vite que ce qu'avait prévu Mott, avec des oscillations, désormais appelées oscillations de Friedel. Outre ce travail, Jacques Friedel suivit à Bristol les séminaires informels de Charles Frank sur les dislocations et les joints de grains, et il lui fit d'ailleurs découvrir le livre Leçons de cristallographie de son grand-père. De retour en région parisienne, J.F. commença par enseigner les dislocations, et ce sont ces cours qui lui ouvrirent la voie vers le poste universitaire qu'il désirait en France. De nombreux mécanismes de plasticité portent son empreinte et son nom.

C'est à Bristol que Jacques connut et épousa Mary Horder, la jeune sœur de Ruth, épouse de Mott. Mary était de dix ans l'aînée de Jacques. Plutôt littéraire et artiste, Mary connaissait déjà fort bien le milieu des physiciens.

Il me semble que, au-delà de sa solide formation physico-mathématique de polytechnicien, la magie de Jacques Friedel consistait à deviner les causes prédominantes pour expliquer nombre d'effets, aussi bien électroniques dans les alliages métalliques avec des hamiltoniens quantiques, que plastiques en métallurgie faisant intervenir les dislocations sans aucun hamiltonien. En cela J.F. ressemblait à Mott et Frank, qu'il appréciait particulièrement. J.F. se gardait bien de faire des calculs complets avec une précision qui aurait dépassé la simplicité de ses hypothèses, et l'accord de ses prédictions avec les effets mesurés le surprenait lui-même. Il travailla aussi sur les disinclinaisons dans les cristaux liquides, et sur les quasi-cristaux. 


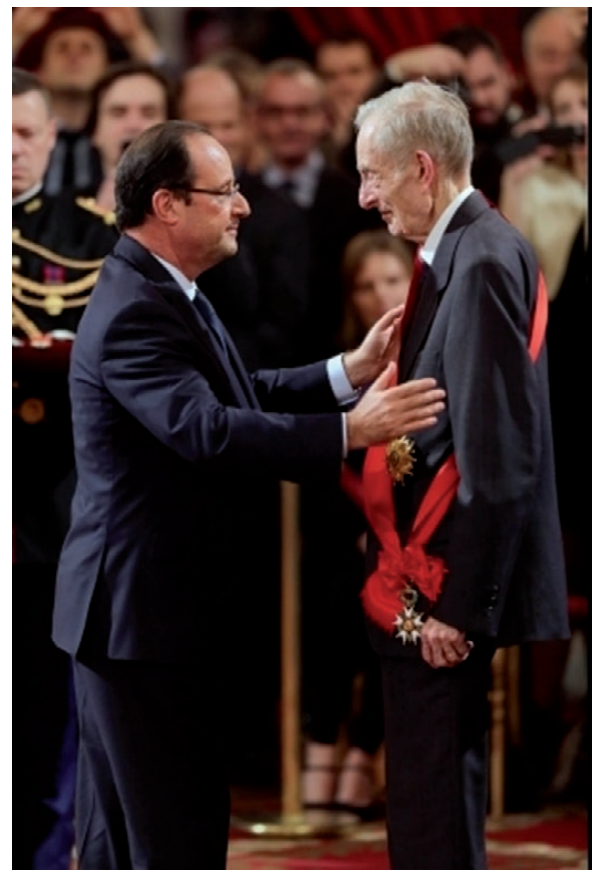

3. Jacques Friedel, lors de la remise de la grand'croix de la Légion d'honneur par le président de la République, François Hollande, le 22 octobre 2013 au Palais de l'Élysée.

Quoique plutôt réservé, il s'impliqua de manière fondamentale dans l'organisation de l'enseignement (physique des solides et des matériaux) et de la recherche en France. Il a présidé la Société française de physique en 1970, la Société Européenne de Physique (EPS) en 1982-84, et l'Académie des sciences en 1993-94. Sa grande culture classique lui permettait de s'intéresser aussi aux problèmes de terminologie scientifique. Jacques fut élevé à la dignité de grand'croix de la Légion d'honneur, le 22 octobre 2013.

\section{Jean et Paul}

Jean, le fils ainé de Jacques et Mary, est médecin dermatologue et dirige un service d'hôpital général en Bourgogne. Il s'est spécialisé dans la généalogie de sa famille. Paul, polytechnicien et docteur d'État en physique des solides, a travaillé en physique des surfaces des semi-conducteurs. Il a eu une carrière industrielle internationale, toujours à l'interface entre la recherche et le marché. Il a présidé la Société de l'électricité, de l'électronique et des technologies de l'information et de la communication (SEE) et dirige aujourd'hui l'École Télécom Bretagne de l'Institut Mines-Télécom. Paul a hérité du violon de son arrière grand-père Georges et il en joue avec talent et plaisir avec ses élèves à Brest, tout comme G.F. en jouait avec ses élèves à Saint-Étienne. I
Ce qui caractérise à nos yeux la Minéralogie et ce qui lui donne son véritable intérêt, c'est précisément qu'elle est le point de rencontre naturel de la Chimie, de la Physique et de la Cristallographie. Le but qu'elle se propose, la détermination complète des minéraux, l'oblige à réagir contre la division extrême que le progrès des Sciences a eu pour conséquence. Cette division, qui permet d'explorer à fond chaque science particulière et d'exploiter ses moindres filons, est cause que l'on néglige trop souvent ces confins où se touchent plusieurs ordres de connaissances et où, comme au contact de deux terrains différents, se trouvent fréquemment accumulées des richesses exceptionnelles.

Charles Friedel, inaugurant sa première leçon à la chaire de minéralogie de la Sorbonne, en 1876

Cité par Stanislao Cannizzaro, chimiste italien (1826-1910), en 1900. On trouve une formule de même esprit dans la préface de son Cours de minéralogie, imprimé en 1893.

\section{Pour aller plus loin}

1- Jacques Friedel, Graine de Mandarin, éditions Odile Jacob (1994).

2 - Les films des interventions faites lors de la Journée intitulée " Les Friedel, la chimie et les cristaux », organisée principalement par l'auteur (OHD) le 22 avril 2014 en l'amphithéâtre Charles Friedel de Chimie ParisTech (Valérie Cabuil, Paul Friedel, Danielle Fauque, Alain Fuchs, Jean-Claude Boulliard, OHD, Didier Nectoux, Robert Mahl, Yves Quéré, Maurice Kléman, François Gautier, Michel Lannoo, Julien Bok, Georges Saada, Ladislas Kubin, Denis Gratias et Yves Bréchet). Interventions filmées par Serge Chiche du CEA et montées par lui en collaboration avec OHD, disponibles sur demande auprès de OHD.

3 $\mathrm{OHD}$, "A review of some elements in the history of grain boundaries, centered on Georges Friedel, the coincident "site' lattice and the twin index", Journal of Materials Science 46 (2011) 4116-4134.

4• OHD, "On the origins of the Finnis-Sinclair potentials", Philosophical Magazine 89 (2009) 3117-3131.

5• OHD, “Charles Crussard's early contributions: Recrystallization in situ and a Grain Boundary study with J. Friedel and B. Cullity”, International Journal of Materials Research (ex Zeitschrift für Metallkunde) 100 (2009) 1382-1388.

6• Jacques Friedel et OHD, “Alan Cottrell, a fundamental metallurgist. In memoriam”, Philosophical Magazine 28-30 (2013) 3703-3713.

7• Yves Bréchet et OHD, « In Memoriam. Disparition de Jacques Friedel », Matériaux et Techniques 102 (2014) No6 (2 pages).

8• Le dossier SFP « Hommage à Jacques Friedel » (Pierre Radvany, Yves Bréchet, Maurice Kléman, Michel Lannoo, François Gautier, Denis Jérome et Jean-Paul Pouget). 16 pages, dossier réalisé à l'initiative des présidents de la Société Française de Physique, Alain Fontaine et Michel Lanno0, à l'automne 2014, téléchargeable à l'adresse www.sfpnet.fr/hommage-a-jacques-friedel.

9• Adrian Sutton et OHD, « Jacques Friedel. 1921-2015 », Biographical Memoirs of Fellows of the Royal Society (2015), en préparation.

10• Les pages 'html' du site www.annales.org/archives/x/. 